${ }^{1}$ L. BRILlouin, Wavepropagation and Group Velocity, Academic Press, London 1960.

2 E. L. INCE, Ordinary Differential Equations, Longmans, Green and Co., Dover 1944.

3 L. D. Landau and E. M. Lifshitz, Electrodynamics of Continuous Media, Pergamon Press, London 1960.
${ }^{4}$ I. S. Nicolis, The Velocity of Energy Transport for Normal Modes Near Total Internal Reflection, Z. Naturforsch. 26 a, 124 [1971].

5 D. J. Kuizenga and A. E. Siegman, FM and AM Mode Locking of the Homogeneous Laser, Part I: Theory, IEEE J. Quantum Electronics, Vol. Q E-6, No. 11, p. 694 [1970].

\title{
Abschätzung von Zustandsdichten des Silbers in $\alpha$-Silber-Mischkristallen mit Gallium, Indium, Germanium und Zinn auf Grund thermodynamischer Untersuchungen mittels galvanischer Festkörperzellen
}

\author{
B. Predel und U. SCHALLNER
}

Institut für Metallforschung der Universität Münster (Westf.)

(Z. Naturforsch. 27 a, 1098-1108 [1972] ; eingegangen am 23. März 1972)

\begin{abstract}
An Estimation of Densities of State of Silver in a-Solid Solutions of Silver with Gallium, Indium, Germanium and Tin based on Thermodynamic Investigations by means of Solid State Galvanic Cells

The partial free enthalpies of formation of the binary $\alpha$-solid solutions of silver with gallium, indium, germanium and tin were determined at $1000^{\circ} \mathrm{K}$ from the EMF of suitable solid state galvanic cells. A further evaluation of the results taking into account known enthalpies of formation yielded the complete partial and integral excess functions of these solid solutions.

An analysis of the difference of the excess values of the partial free enthalpies of formation of the components showed that the lattice distortion, due to differences in atomic radii of the solid solutions studied, is not very significant. Differences in the structure between the solid solution and its components can be clearly noted only in the case of the silver-germanium solid solutions. The energetics of the alloys are determined principally by the filling of the $5 \mathrm{~s}^{1}$ conduction band of the silver by the electrons contributed by higher-valent constituents. From the thermodynamic results the density of state of the conduction band of the silver was estimated to be smaller than 0.31 electr. per atom and $\mathrm{eV}$. The density of state increases with increasing concentration of the alloying component; this can be explained by a change in the Fermi surface in the silver host lattice.
\end{abstract}

\section{Einführung}

In zahlreichen neueren Untersuchungen wird der Frage nachgegangen, welche Faktoren in einem individuellen metallischen Zweistoffsystem für die energetischen Verhältnisse der jeweiligen Mischphasen verantwortlich sind. Es hat sich dabei als zweckmäßig erwiesen, die Überschußfunktionen als in einfacher Weise additiv aus Einzelanteilen zusammengesetzt zu betrachten, die von den jeweiligen einzelnen Einflußgrößen herrühren. In der Regel werden dabei drei verschiedene Einflüsse betrachtet, die zweifellos nur modellmäßig scharf voneinander getrennt werden können ${ }^{1-3}$. Von wesentlicher Bedeutung ist in vielen Fällen die Änderung der chemischen Bindungsverhältnisse bei der Legierungsbildung. Daneben kann im Falle großer Atomradiendifferenzen der Legierungspartner auch die Gitterverzerrung in festen Mischphasen von Bedeutung

Sonderdruckanforderungen an Prof. Dr. B. PREDEL, Institut für Metallforschung, Westf. Wilhelms-Universität, D-4400 Münster (Westf.), Steinfurter Straße 107. sein. Schließlich können sich noch strukturelle Unterschiede auf die energetischen Eigenschaften einer Legierung auswirken, wenn die betrachtete Mischphase nicht isotyp ist mit den Gitterstrukturen der Partner.

Zur Abschätzung der Gitterverzerrungseinflüsse sind an Hand von Modellen Näherungsrechnungen entwickelt ${ }^{4,5}$ sowie empirische Methoden zur Erschließung von Fehlpassungseinflüssen aufgezeigt worden ${ }^{6}$. Der strukturelle Einfluß, der sogenannte Umwandlungsanteil, kann durch Auswertung experimentell ermittelter Mischungsenthalpien und Überschußentropien entsprechender Systeme ermittelt werden ${ }^{3,7}$. Eine vollständige Klärung des „Bindungsanteils" ist indessen bisher nur in wenigen Fällen in quantitativer Weise gelungen. Das ist bei der Vielzahl an individuellen Möglichkeiten der oft recht komplexen Bindungsverhältnisse in metallischen Systemen nicht verwunderlich.

Bei einer großen Anzahl von Legierungen kann bekanntlich eine Reihe physikalischer Eigenschaften an Hand des Bändermodells erklärt werden. Es er- 
scheint von Interesse, solche Legierungen unter diesem Gesichtspunkt auch hinsichtlich ihrer thermodynamischen Eigenschaften zu betrachten. Einige diesbezügliche Untersuchungen sind bereits durchgeführt worden ${ }^{2,8-11}$. Beispielsweise konnte gezeigt werden, daß nach Eliminierung des Gitterverzerrungseffektes der verbleibende Rest der thermodynamischen Überschußfunktionen von Palladium - Wasserstoff-Einlagerungsmischkristallen allein durch die Auffüllung des 4d- und 5s-Bandes des Palladiums durch die vom Wasserstoff abgegebenen Elektronen festgelegt ist. Auch bei den Substitutionsmischkristallen des Systems Palladium - Silber liegen ähnliche Verhältnisse vor ${ }^{2}$. Beim Ersatz des Silbers durch höherwertige Elemente derselben Periode des Periodensystems sind beträchtliche Werte für die „elektronische freie Mischungsenthalpie“ zu erwarten. Untersuchungen von BRODOWSKY und SCHALLER ${ }^{11}$ haben dies an einigen Palladium-Legierungen bestätigt. Gleichzeitig ist indessen gefunden worden, daß neben einer Bandauffüllung offenbar infolge des Einbaus fremder Ionenrümpfe in das Gitter eine Änderung der Bandstruktur eintreten kann.

Entsprechende Verhältnisse sind bei Mischkristallen des Kupfers, Silbers und Goldes zu erwarten, und zwar insbesondere mit Elementen, die mit diesen IB-Metallen bei den jeweils betreffenden Valenzelektronen-Konzentrationen Hume-Rothery-Verbindungen bilden und dadurch bereits den dominierenden Einfluß der freien Elektronen auf die Stabilität der Legierungen dieser Systeme dokumentieren. Um sowohl die Auswirkung der Wertigkeit als auch die unterschiedlicher Fremdionenrümpfe zu erfassen, ist zunächst die Ermittlung einiger thermodynamischer Zustandsgrößen von Legierungen des Silbers mit Gallium, Indium, Germanium und Zinn in Angriff genommen worden. Als experimentelle Methode wurde die Ermittlung der partiellen freien Bildungsenthalpien der unedleren Legierungskomponente nach der EMK-Methode mit festen Elektrolyten gewählt. Sie liefert insbesondere bei den speziell interessierenden silberreichen Legierungen Resultate hoher Genauigkeit.

\section{Experimentelle Durchführung}

Die Untersuchungen wurden mit einer galvanischen Zelle durchgeführt, wie sie erstmals von RAPP und MAAK ${ }^{8}$ für die Ermittlung partieller freier Bildungsenthalpien fester Kupfer - Nickel-Mischphasen angewandt worden ist. Als fester Elektrolyt diente ein Oxid mit Sauerstoffionen-Leitung - eine Möglichkeit, die bereits von KIUkKola und WAGNER ${ }^{12}$ mit Erfolg zur Bestimmung der freien Bildungsenthalpien einiger Metalloxide genutzt worden ist. Eine solche Festkörperkette kann schematisch folgenden Aufbau haben:

$$
\begin{aligned}
& \oplus\left|\mathrm{A}+\mathrm{A}_{2} \mathrm{O}_{z}\right| \text { fester } \mathrm{O}^{-2} \text {-Leiter } \mid \\
& \mathrm{A}-\mathrm{B} \text {-Legierung }+\mathrm{A}_{2} \mathrm{O}_{z} \mid \ominus
\end{aligned}
$$

$\mathrm{A}_{2} \mathrm{O}_{z}$ ist das stabile Oxid der Komponente A (hier $\mathrm{Ga}, \mathrm{In}, \mathrm{Ge}$ und $\mathrm{Sn}$ ) und $z$ ihre Wertigkeit. Rechts und links vom Elektrolyten stellen sich bestimmte Sauerstoffpartialdrucke ein. Der Druckausgleich kann durch den sauerstoffionenleitenden Elektroly. ten erfolgen. Bei isothermer und reversibler Versuchsführung ist die dabei eintretende Änderung der partiellen freien Enthalpie des Sauerstoffs aus der EMK $(E)$ ermittelbar. Über den durch das Massenwirkungsgesetz gegebenen Zusammenhang zwischen den Sauerstoff-Partialdrucken und dem Partialdruck der Komponente $\mathrm{A}$ ist auch ihre partielle freie Bildungsenthalpie gegeben, und zwar gemäß:

$$
\overline{\Delta G}_{\mathrm{A}}=-z F E \text {; }
$$

$F$ bedeutet die Faraday-Konstante.

Der in Gl. (1) wiedergegebene einfache Aufbau einer Festkörperzelle konnte bei unseren Untersuchungen nicht realisiert werden, weil die als unedlere Komponente der Silber-Mischkristalle vorgesehenen Elemente bei den für die Gleichgewichtseinstellung der Zelle erforderlichen hohen Temperaturen nicht mehr im festen Zustand vorliegen. Als Bezugselektrode wurde daher an Stelle von $\left|\mathrm{A}+\mathrm{A}_{\mathbf{2}} \mathrm{O}_{z}\right|$ eine $|\mathrm{Ni}+\mathrm{NiO}|$-Elektrode eingesetzt:

$$
\begin{array}{r}
|\mathrm{Ni}+\mathrm{NiO}| 0,85 \mathrm{ThO}_{2}+0,15 \mathrm{Y}_{2} \mathrm{O}_{3} \mid \\
\mathrm{A}-\mathrm{B} \text {-Legierung }+\mathrm{A}_{2} \mathrm{O}_{z} \mid .
\end{array}
$$

Die mit der Zelle (3) bestimmten $\overline{\Delta G}_{\mathrm{A}}$-Werte können in einfacher Weise in die entsprechenden Werte der Zelle vom Typ (1) umgerechnet werden, wenn die freien Bildungsenthalpien der Oxide $\mathrm{A}_{2} \mathrm{O}_{z}$ und des $\mathrm{NiO}$ bekannt sind. Werden die freien Bildungsenthalpien dieser Oxide jeweils auf 1 Mol Sauerstoff bezogen, so gilt

$$
E=E^{0}+\left(\Delta G_{\mathrm{NiO} O}-\Delta G_{\mathrm{A}_{z} \mathrm{O}_{z}}\right) / 4 F .
$$

$E$ bedeutet die für die Zelle (1) zu erwartende und $E^{0}$ die mit der Zelle (3) gemessene EMK. Als Elektrolyt wurden Sinterkörper aus $85 \mathrm{Mol}^{-} \% \mathrm{ThO}_{2}$ und $15 \mathrm{Mol}-\% \mathrm{Y}_{2} \mathrm{O}_{3}$ verwendet. 
Ein Ziel dieser Untersuchungen ist die Abschätzung der Zustandsdichte des 5s-Bandes des Silbers an der Fermi-Grenze und ihre eventuelle Beeinflussung durch Legierungszusätze. Um sie zu ermitteln, ist eine genaue Kenntnis des Steigmaßes der $\overline{\Delta G}_{\mathrm{A}}-x_{\mathrm{A}}$-Kurven erforderlich. Auch die Ermittlung des für die Behandlung von Diffusionsproblemen notwendigen thermodynamischen Faktors $\mathrm{d} \ln a_{\mathrm{A}} / \mathrm{d} \ln x_{\mathrm{A}}$ (mit $a_{\mathrm{A}}=$ thermodynamische Aktivität und $x_{\mathrm{A}}=$ Molenbruch der Komponente A) erfolgt unmittelbar aus der Konzentrationsabhängigkeit der partiellen freien Mischungsenthalpien. Es ist daher sinnvoll, die Steigung der $\overrightarrow{\Delta G}_{\mathrm{A}}-x_{\mathrm{A}}-$ Kurven möglichst in einem direkten Experiment zu ermitteln. Das kann mit Hilfe folgender galvanischer Kette erfolgen:

$$
\begin{array}{r}
\mid \mathrm{A}_{x_{1}}-\mathrm{B}_{\left(1-x_{1}\right)}-\text { Leg. }+\mathrm{A}_{2} \mathrm{O}_{z}\left|0,85 \mathrm{ThO}_{2}+0,15 \mathrm{Y}_{2} \mathrm{O}_{3}\right| \\
\mathrm{A}_{x_{2}}-\mathrm{B}_{\left(1-x_{2}\right)}-\text { Leg. }+\mathrm{A}_{2} \mathrm{O}_{z} \mid .
\end{array}
$$

Durch Wahl eng benachbarter Legierungskonzentrationen $x_{1}$ und $x_{2}$ der Komponente A kann das Steigmaß der $\overline{\Delta G}_{\mathrm{A}}-x_{\mathrm{A}}$-Funktion relativ genau unmittelbar bestimmt werden. Ein besonderer Vorteil dabei besteht ferner darin, daß die in der Kette (3) für die Absolutbestimmung der $\overline{\Delta G}_{\mathrm{A}}$-Werte erforderliche Hilfselektrode $|\mathrm{Ni}+\mathrm{NiO}|$ entfällt und damit Unsicherheiten, die durch eine nicht ausreichend genaue Kenntnis der Werte für die freien Bildungsenthalpien von $\mathrm{NiO}$ und $\mathrm{A}_{2} \mathrm{O}_{z}$ in das Endresultat bei der Auswertung eingeschleppt werden können, hier nicht auftreten.

Die Versuchsanordnung ist in Abb. 1 wiedergegeben. Sie entspricht im Prinzip derjenigen, die SchmalzRIED ${ }^{13}$ bzw. RAPP und MAAK ${ }^{8}$ für ähnliche Untersuchungen benutzt haben. Die Festkörperkette war in einem keramischen Rohr (Pythagorasmasse) untergebracht. Die Pillen der Zelle wurden mit einer verstellbaren Schraube und einer Spiralfeder durch ein Pythagoras-Röhrchen gegen einen keramischen Block gepreßt. Die elektrischen Kontakte bestanden aus Platin. Um das störende Auftreten induzierter Spannungen zu verhindern, wurde über das die Versuchsanordnung vakuumdicht abschließende, äußere Pythagorasrohr eine geerdete Platinfolie gelegt. Diese Versuchsanordnung befand sich in einem Röhrenofen. Die Versuche wurden in einer Atmosphäre aus Reinstargon (99,995\% Ar) durchgeführt. Auf eine weitere Reinigung des Schutzgases konnte verzichtet werden, nachdem nachgewiesen war, daß bei Verwendung von Argon mit der genannten Reinheit im allgemeinen keine Abhängigkeit der EMK-Werte von der Strömungsgeschwindigkeit des Schutzgases auftrat und außerdem eine zusätzliche Reinigung des Argons mit einem aktivierten Molekularsieb aus Silicagel mit aufgebrachtem Chromtrioxid (Sauerstoff $<0,1 \mathrm{vpm}$ und Wasserdampf $<0,5 \mathrm{vpm})$ keine erkennbare Verbesserung erbrachte. Die EMK wurde mit einem hochohmigen TransistorVoltmeter gemessen. Sein Eingangswiderstand von $100 \mathrm{M} \Omega$ gewährleistete eine praktisch stromlose Spannungsmessung. Die Temperatur der Festkörperzelle wurde mit einem geeichten $\mathrm{Pt}-\mathrm{Pt} / \mathrm{Rh}$-Thermoelement auf $\pm 1^{\circ}$ genau gemessen.

Zur Herstellung der einzelnen Metall-Oxid-Elektroden dienten Materialien hoher Reinheit. Eine Übersicht über die Ausgangsmaterialien gibt Tabelle 1. Die Legierungen wurden in Korund-Tiegeln unter Argon als Schutzgas erschmolzen und wenig unterhalb der jeweiligen Solidustemperatur im Vakuum solange geglüht, bis sie völlig homogen waren. Die erforderlichen Temperzeiten lagen in der Größenordnung von 20 bis 30 Tagen. Feilspäne dieser Legierungen wurden mit

\begin{tabular}{ll}
\hline Substanz & Reinheit \\
\hline $\mathrm{Ni}$ & $99,99 \% \mathrm{Ni}$ \\
$\mathrm{Ag}$ & $\mathbf{9 9 , 9 9 5 \% \mathrm { Ag }}$ \\
$\mathrm{Ga}$ & $\mathbf{9 9 , 9 9 9} \% \mathrm{Ga}$ \\
$\mathrm{In}$ & $\mathbf{9 9 , 9 9 9} \% \mathrm{In}$ \\
$\mathrm{Ge}$ & $\mathrm{a}$ \\
$\mathrm{NiO}$ & reinst \\
$\mathrm{Ga}_{2} \mathrm{O}_{3}$ & $\mathbf{9 9 , 9 9} \% \quad \mathrm{Ga}_{2} \mathrm{O}_{3}$ \\
$\mathrm{In}_{2} \mathrm{O}_{3}$ & reinst \\
$\mathrm{GeO}_{2}$ & $99,999 \% \mathrm{GeO}_{2}$ \\
$\mathrm{SnO}_{2}$ & rein \\
&
\end{tabular}

Tab. 1. Reinheit der eingesetzten Substanzen.

a Durch Zonenschmelzen hochgereinigt; spezifischer elektrischer Widerstand $30 \Omega \cdot \mathrm{cm}$.

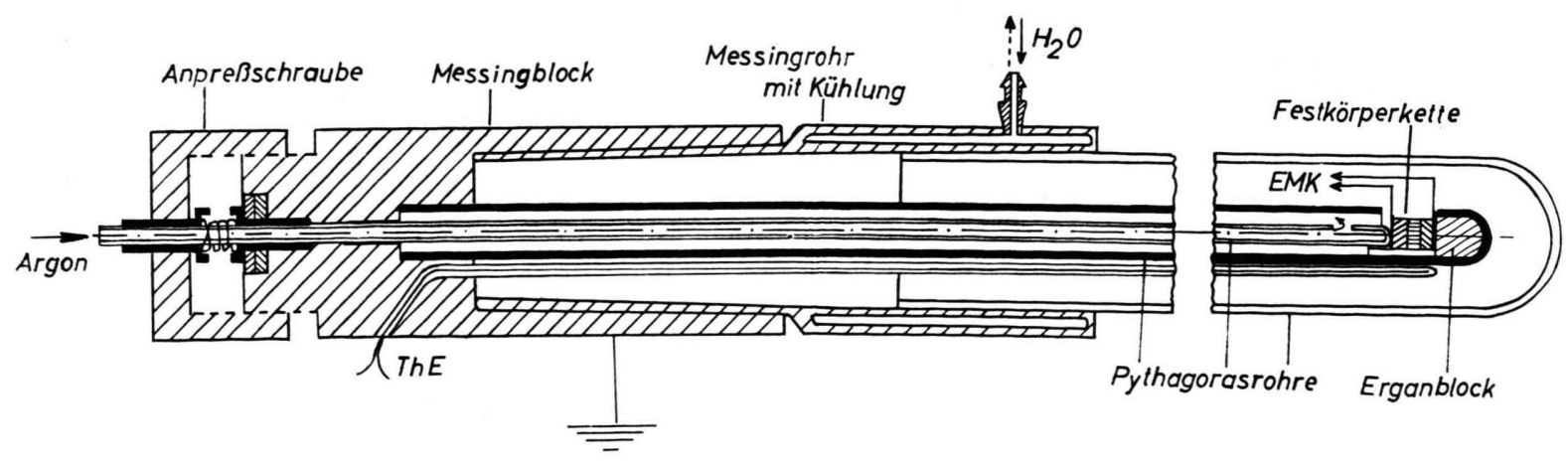

Abb. 1. Versuchsanordnung zur Untersuchung fester Mischphasen nach der EMK-Methode. 
den entsprechenden pulverförmigen Oxiden im Gewichtsverhältnis von etwa $3: 1$ vermischt und zu flachen zylinderförmigen Pillen gepreßt. In gleicher Weise wurde auch die $\mathrm{Ni}-\mathrm{NiO}$-Bezugselektrode hergestellt.

Vor Beginn der Messungen wurde die Festkörperzelle zur Einstellung des thermodynamischen Gleichgewichts 10 bis 15 Stunden bei einer Temperatur gehalten, die wenig unterhalb der Solidustemperatur der eingesetzten Legierung lag. Erst nach einer solchen Vorbehandlung konnten zeitlich konstante EMK-Werte erhalten werden. Im Verlaufe einer Meßreihe wurde dann die Temperatur stufenweise reduziert und jeweils abgewartet, bis der neue EMK-Wert konstant blieb. Die Dauer bis zur Einstellung des Gleichgewichts nimmt naturgemäß mit sinkender Temperatur zu. Bei $700^{\circ} \mathrm{C}$ betrug sie bereits mehrere Stunden. Unterhalb dieser Temperatur wurden keine Messungen durchgeführt. Die Reversibilität der Gleichgewichtseinstellung wurde durch Messung der EMK-Werte bei fallender und steigender Temperatur überprüft.

\section{Experimentelle Ergebnisse}

\section{Kontrolle der Meßanordnung}

Zur Prüfung der Leistungsfähigkeit der aufgebauten Versuchsanordnung wurde die EMK der Festkörperkette

$$
\begin{aligned}
\oplus|\mathrm{Ni}+\mathrm{NiO}| 0,85 \mathrm{ThO}_{2} \\
+0,15 \mathrm{Y}_{2} \mathrm{O}_{3}\left|\mathrm{Cu}+\mathrm{Cu}_{2} \mathrm{O}\right| \ominus
\end{aligned}
$$

ermittelt, für die in der Literatur ${ }^{12,14}$ Vergleichswerte vorliegen. Die erzielten Ergebnisse sind in Tab. 2 den Literaturdaten gegenübergestellt. Die Abweichungen von den bisher bekannten EMK-Werten der Festkörperkette (6) betragen maximal 4 bzw. $9 \mathrm{mV}$. Umgerechnet auf einen relativen Fehler für die freie Bildungsenthalpie des $\mathrm{NiO}$ bedeutet diese Abweichung einen maximalen Fehler von $1 \%$. Diese gute Übereinstimmung zeigt die Zuverlässigkeit der Versuchsanordnung.

Tab. 2. EMK-Werte der galvanischen Kette (6).

\begin{tabular}{llllll}
\hline \multicolumn{7}{c}{$\begin{array}{c}\text { EMK-Werte in } \mathrm{mV} \text { bei } \\
700^{\circ} \mathrm{C}\end{array}$} & $800^{\circ} \mathrm{C}$ & $900^{\circ} \mathrm{C}$ & $1000^{\circ} \mathrm{C}$ & $1050^{\circ} \mathrm{C}$ \\
\hline $\begin{array}{l}\text { KIUKKoLA u. } \\
\text { WAGNER }^{12}\end{array}$ & - & 266 & 263 & 257 & 254 \\
$\begin{array}{l}\text { STEELE und } \\
\begin{array}{l}\text { ALCOCK } \\
\text { eigene Werte }\end{array}\end{array}$ & 280 & 273 & 266 & 259 & - \\
\hline
\end{tabular}

\section{Messungen an Festkörperzellen mit Silber-Legierungen}

Es wurden sowohl Absolutmessungen mit einer Zelle vom Typ (3) als auch Relativmessungen mit einer Zelle vom Typ (5) durchgeführt. Die mit einer Zelle (3) erhaltenen EMK-Werte $E^{0}$ wurden mit Hilfe der Gl. (4) in EMK-Werte $E$ einer Zelle (1) umgerechnet. Die dabei benötigten Werte für die freie Bildungsenthalpie der Nickel-, Gallium-, Indium-, Germanium- und Zinn-Oxide $\left(\Delta G_{\mathrm{A}_{2} \mathrm{O}_{z}}\right)$ wurden Literaturangaben ${ }^{15}$ entnommen (siehe Tab. 4). Die Ergebnisse $\Delta E$ mittels einer Zelle (5) bestätigten im allgemeinen die aus Absolutmessungen erhaltenen $E$-Werte. Dort, wo sich unterschiedliche $E$ - bzw. $\left(\mathrm{d} E / \mathrm{d} x_{\mathrm{A}}\right)$-Werte ergaben, was in manchen Fällen bei kleinen A-Konzentrationen auftrat, sind die aus Relativmessungen erhaltenen Ergebnisse denen aus Absolutmessungen vorgezogen worden. Die so erhaltenen $E$-Werte sind in Tab. 3 zusammengestellt.

Tab. 3. Ergebnisse der EMK-Messungen an festen Silber-

\begin{tabular}{|c|c|c|c|}
\hline$x_{\mathrm{Ga}}$ & $E$ & $x_{\text {In }}$ & $E$ \\
\hline 0,01 & 603 & 0,01 & 481 \\
\hline 0,02 & 568 & 0,02 & 442 \\
\hline 0,04 & 509 & 0,05 & 358 \\
\hline 0,06 & 465 & 0,10 & 259 \\
\hline 0,08 & 432 & 0,15 & 164 \\
\hline 0,10 & 415 & 0,18 & 114 \\
\hline$x_{\mathrm{Ge}}$ & & $x_{\mathrm{Sn}}$ & \\
\hline 0,01 & 380 & 0,01 & 386 \\
\hline 0,02 & 335 & 0,02 & 355 \\
\hline 0,03 & 287 & 0,04 & 312 \\
\hline 0,04 & 245 & 0,06 & 289 \\
\hline 0,05 & 220 & 0,08 & 270 \\
\hline 0,06 & 193 & 0,10 & 259 \\
\hline 0,07 & 169 & 0,11 & 248 \\
\hline
\end{tabular}
Mischphasen bei $1000^{\circ} \mathrm{K}$. EMK-Werte in $\mathrm{mV}$.

\section{Betrachtung einiger Störmöglichkeiten}

Damit die EMK-Werte, die sich an einer Festkörperzelle des Typs (3) oder (5) einstellen, eindeutig ausgewertet werden können, muß gewährleistet sein, daß allein die Überführung von Sauerstoffionen von der einen zur anderen Elektrode den Meßeffekt hervorruft. Nach Hund und MEZger ${ }^{16}$ ist dies bei Verwendung der $\left(\mathrm{ThO}_{2}+\mathrm{Y}_{2} \mathrm{O}_{3}\right)$-Mischphase als Elektrolyt gewährleistet. Diese Oxidmischkristalle besitzen ein vollkommenes Kationenteilgitter mit mehr oder weniger Leerstellen im Anionenteilgitter. Bei hohen Temperaturen sind die Leerstellen hinreichend beweglich und bedingen eine $\mathrm{O}^{2-}$-Leitfähigkeit. Daneben muß noch bei hohen Sauerstoffpartialdrucken mit einer zusätzlichen De- 
fektelektronenleitung und bei sehr niedrigen Sauerstoffdrucken mit einer Überschußelektronenleitung gerechnet werden. Nach Untersuchungen von STEELE und Alcock ${ }^{14}$ tritt bei $1000{ }^{\circ} \mathrm{C}$ bei Sauerstoffpartialdrucken zwischen $10^{-6}$ und $10^{-25}$ atm reine Ionenleitung auf. LIDSTER und BELL ${ }^{17}$ geben den Bereich der reinen Ionenleitung noch größer an, nämlich zwischen $10^{-4}$ und $10^{-27,5}$ atm Sauerstoff. Mit steigender Temperatur zwischen $800^{\circ} \mathrm{C}$ und $1100{ }^{\circ} \mathrm{C}$ wird dieser Druckbereich nur geringfügig enger.

Tab. 4. Freie Bildungsenthalpien von Oxiden in kcal/Mol Sauerstoff und ihre Sauerstoffpartialdrucke in atm bei $1000^{\circ} \mathrm{K}$.

\begin{tabular}{lll}
\hline Oxid & $\Delta G_{\text {Oxid }}{ }^{15}$ & $\left(-\log p_{\mathrm{O}_{2}}\right)$ \\
\hline $\mathrm{ThO}_{2}$ & $-246,6$ & 53,8 \\
$\mathrm{YO}_{3 / 2}$ & $-231,1$ & 50,5 \\
$\mathrm{Ga}_{2} \mathrm{O}_{3}$ & $-118,0$ & 25,8 \\
$\mathrm{In}_{2} \mathrm{O}_{3}$ & $-95,3$ & 20,8 \\
$\mathrm{SnO}_{2}$ & $-89,1$ & 19,4 \\
$\mathrm{SnO} \mathrm{O}$ & $-87,2$ & 19,0 \\
$\mathrm{GeO}_{2}$ & $-87,0$ & 19,0 \\
$\mathrm{NiO}$ & $-70,2$ & 15,3 \\
$\mathrm{Cu} O$ & $-46,4$ & 10,1 \\
$\mathrm{CuO}$ & $-32,4$ & 7,1 \\
$\mathrm{Ag}_{2} \mathrm{O}$ & $+37,7$ & - \\
\hline
\end{tabular}

Tabelle 4 gibt einen Überblick über die Sauerstoffpartialdrucke der bei unseren Experimenten verwendeten Oxide. Die $\mathrm{O}_{2}$-Partialdrucke wurden aus den bekannten freien Bildungsenthalpien ${ }^{15}$ der Oxide berechnet. Ein Blick auf Tab. 4 zeigt, daß sämtliche aufgeführten Partialdruckwerte des Sauerstoffs innerhalb des von LIDSTER und BELL ${ }^{17}$ angegebenen Intervalls für reine Ionenleitung liegen. Die Voraussetzungen für die Auswertung der EMKWerte gemäß Gl. (2) sind erfüllt.

Damit sich keine Mischpotentiale in der Zelle einstellen, dürfen keine Austauschreaktionen zwischen dem Elektrolyt und den Elektroden stattfinden. Wäre ein irreversibler Stoffaustausch durch die entsprechenden Grenzflächen vorhanden, dann würden die auftretenden Konzentrationsgradienten zu einer nicht einfach überschaubaren Änderung der Überführungseigenschaften der Sauerstoffionen in der Zelle führen. Solche Austauschreaktionen treten praktisch nicht auf, wenn für die freien Bildungsenthalpien der beteiligten Oxide gilt:

$$
\begin{gathered}
\Delta G_{\mathrm{NiO}} \gg \Delta G_{\mathrm{ThO} O_{2}} \text { bzw. } \Delta G_{\mathrm{Y}_{2} \mathrm{O}_{3}} \\
\text { und } \\
\Delta G_{\mathrm{B}_{2} \mathrm{O}_{z}} \gg \Delta G_{\mathrm{A}_{2} \mathrm{O}_{z}} \gg \Delta G_{\mathrm{ThO} z} \text { bzw. } \Delta G_{\mathrm{Y}_{2} \mathrm{O}_{3}} .
\end{gathered}
$$

Dabei bedeutet $\mathrm{B}$ die edlere und $\mathrm{A}$ die unedlere Komponente der Legierung. Wie aus Tab. 4 ersichtlich, ist diese Bedingung bei den von uns verwendeten Systemen gut erfüllt, so daß Störungen durch Nebenreaktionen auszuschließen sind.

Fehler können schließlich auftreten, wenn die eingesetzten Sauerstoffverbindungen aus Gemischen verschiedener Oxide bestehen. Nur beim Zinnoxid und Kupferoxid könnte mit einer solchen Störung gerechnet werden. Die $\Delta G$-Werte der entsprechenden Oxide in Tab. 4 zeigen jedoch, daß nur mit den Oxiden $\mathrm{SnO}_{2}$ bzw. $\mathrm{Cu}_{2} \mathrm{O}$ als den stabilen Verbindungen zu rechnen ist.

\section{Auswertung}

Die aus den experimentell ermittelten $E^{0}$.Werten berechneten $E$-Werte der Festkörperzelle des Typs (1), die hinsichtlich ihrer Konzentrationsabhängigkeit an Hand der mit der Kette (5) gewonnenen $\Delta E$-Werte kontrolliert und korrigiert worden sind, lieferten zunächst gemäß Gl. (2) partielle freie Bildungsenthalpien $\left(\overline{\Delta G}_{\mathrm{A}}\right)$ der jeweils unedleren Legierungskomponente. Für die weiteren Betrachtungen sind die Überschußwerte der partiellen freien Bildungsenthalpien

$$
\overline{\Delta G}_{\mathrm{A}}^{\mathrm{ex}}=\overline{\Delta G}_{\mathrm{A}}-R T \ln x_{\mathrm{A}}
$$

von besonderem Interesse. $R$ ist die Gaskonstante. Die Überschußwerte der partiellen freien Bildungsenthalpien des Silbers sind mit Hilfe der DuhemMargules-Gleichung aus der Konzentrationsabhängigkeit der entsprechenden Werte der unedleren Komponente A berechnet worden:

$$
\begin{gathered}
\overline{\Delta G}_{\mathrm{Ag}}^{\mathrm{ex}}=\int_{0}^{x_{\mathrm{A}}} \alpha_{\mathrm{A}} \mathrm{d} x_{\mathrm{A}}-\alpha_{\mathrm{A}} x_{\mathrm{A}}\left(1-x_{\mathrm{A}}\right) \\
\alpha_{\mathrm{A}}:=\frac{\overline{\Delta G}_{\mathrm{A}}^{\mathrm{ex}}}{\left(1-x_{\mathrm{A}}\right)^{2}} .
\end{gathered}
$$

Mit

$$
\Delta G^{\mathrm{ex}}=x_{\mathrm{A}} \overline{\Delta G}_{\mathrm{A}}^{\mathrm{ex}}+\left(1-x_{\mathrm{A}}\right) \overline{\Delta G}_{\mathrm{Ag}}^{\mathrm{ex}}
$$

sind schließlich in bekannter Weise die integralen freien Bildungsenthalpien der Silberlegierungen berechnet worden. Ferner sind noch nach

$$
\overline{\Delta G}_{\mathrm{A}}=R T \ln a_{\mathrm{A}}
$$

die thermodynamischen Aktivitäten $a$ der Komponenten und, soweit zugänglich, unter Verwendung kalorimetrisch bestimmter Werte für die Bildungs- 
enthalpien $\Delta H$ gemäß

$$
\Delta G^{\mathrm{ex}}=\Delta H-T \Delta S^{\mathrm{ex}}
$$

die Überschußwerte der integralen Bildungsentropien $\Delta S$ berechnet worden. Die resultierenden Zahlenwerte sind in Tab. 5 und 6 zusammengestellt. Die Uberschußwerte der partiellen freien Bildungsenthalpien der unedleren Komponenten, die also unmittelbar aus den Meßdaten resultierenden Größen, sind in den Abb. 2 und 3 als Funktion der Legierungskonzentration wiedergegeben.

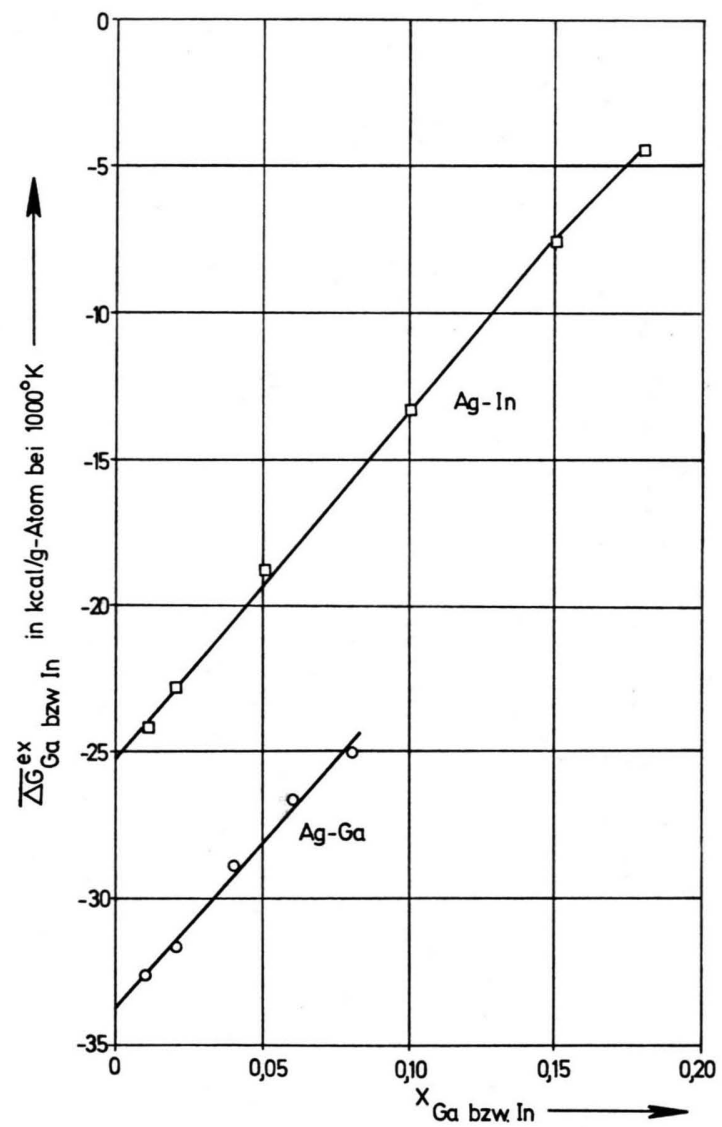

Abb. 2. Uberschußwerte der partiellen freien Bildungsenthalpie von $a$-Silber-Mischkristallen mit Gallium und Indium bei $1000^{\circ} \mathrm{K}$.

\section{Diskussion der Ergebnisse}

\section{Aufgliederung der partiellen Überschußfunktionen in Einzelanteile}

Da im vorliegenden Falle der $\alpha$-Mischkristalle des Silbers mit den höherwertigen Metallen Gallium, Indium, Germanium und Zinn weder die Atom-

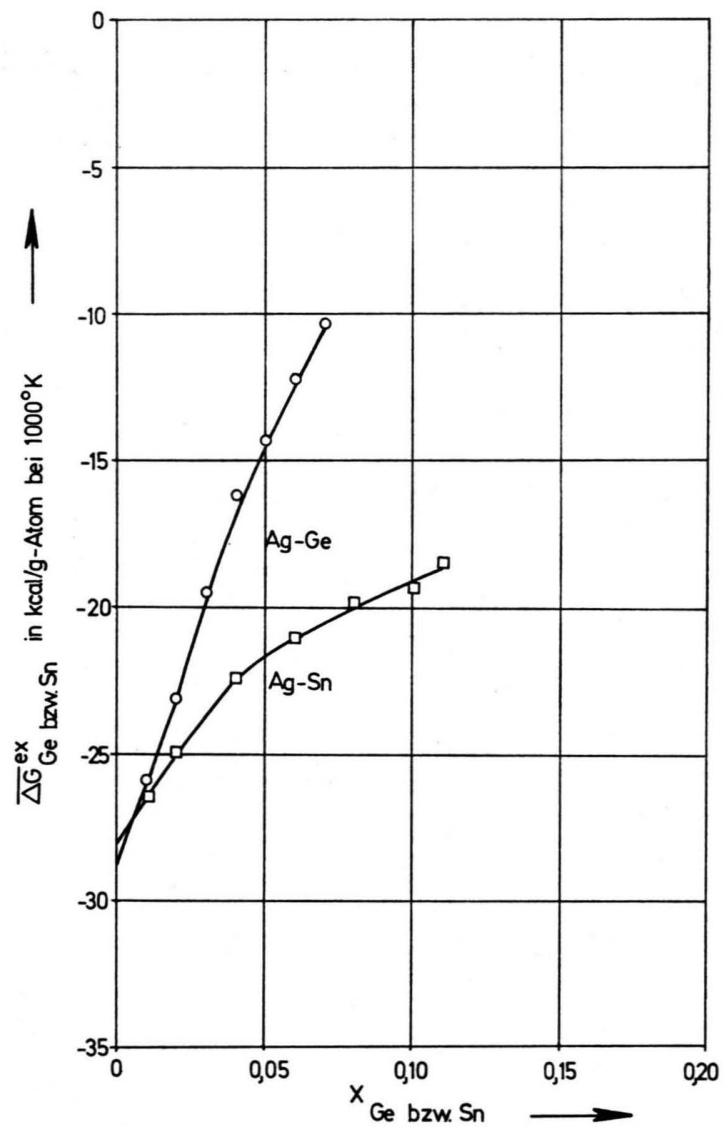

Abb. 3. Uberschußwerte der partiellen freien Bildungsenthalpie von $\alpha$-Silber-Mischkristallen mit Germanium und Zinn bei $1000^{\circ} \mathrm{K}$.

radien noch die Gitterstrukturen der jeweiligen Komponenten gleich sind und zudem mit einer Änderung der chemischen Bindungsverhältnisse bei der Legierungsbildung $\mathrm{zu}$ rechnen ist, muß angenommen werden, daß das Vorzeichen und der Betrag der experimentell ermittelten thermodynamischen $\mathrm{Zu}$ standsgrößen durch das Zusammenwirken eines Bindungsanteils, eines Fehlpassungsanteils und eines Strukturumwandlungsanteils bedingt sind. Für die beiden letztgenannten Einflußgrößen sind positive Beiträge zu den Überschußfunktionen zu erwarten. Das negative Vorzeichen der Überschußwerte der partiellen freien Bildungsenthalpien weist daher auf einen dominierenden Einfluß des Bindungsanteils hin. Um bei der Legierungsbildung auftretende Änderungen der elektronischen Verhältnisse näher untersuchen zu können, ist es zweckmäßig, die beiden anderen Anteile entweder zu eliminieren oder nachzuweisen, daß ihr Einfluß gegenüber dem elektronischen Bindungsanteil vernachlässigbar ist. 
Tab. 5. Thermodynamische Bildungsdaten fester $\alpha$-Mischphasen des Silber-Gallium- und Silber - Indium-Systems bei $1000^{\circ} \mathrm{K}$. $\Delta G$ - und $\Delta H$-Werte in $\mathrm{kcal} / \mathrm{g}$-At om und $\Delta S$-Werte in $\mathrm{cal} / \mathrm{g}$-Atom ${ }^{\circ} \mathrm{K}$.

\begin{tabular}{|c|c|c|c|c|c|c|c|}
\hline$x_{\mathrm{Ga}}$ & $\overline{\Delta G}_{\mathrm{Ga}}^{\mathrm{ex}}$ & $\overline{\Delta G}_{\mathrm{Ag}}^{\mathrm{ex}}$ & $\Delta G^{\mathrm{ex}}$ & $a_{\mathrm{Ga}}$ & $a_{\mathrm{Ag}}$ & $\Delta H^{18}$ & $\Delta S^{\mathrm{ex}}$ \\
\hline 0,01 & $-32,58$ & 0 & $-0,33$ & $7 \cdot 10^{-10}$ & 0,99 & & \\
\hline 0,02 & $-31,54$ & $-0,09$ & $-0,72$ & $3 \cdot 10^{-9}$ & 0,94 & & \\
\hline 0,04 & $-28,83$ & $-0,03$ & $-1,18$ & $2 \cdot 10^{-8}$ & 0,94 & $-0,04$ & $+1,14$ \\
\hline 0,06 & $-26,59$ & $-0,21$ & $-1,79$ & $9 \cdot 10^{-8}$ & 0,85 & & \\
\hline 0,08 & $-24,88$ & $-0,34$ & $-2,30$ & $2,9 \cdot 10^{-7}$ & 0,78 & $-0,05$ & $+2,25$ \\
\hline 0,10 & $-22,50$ & $-0,57$ & $-2,76$ & $1,2 \cdot 10^{-6}$ & 0,68 & & \\
\hline$x_{\text {In }}$ & $\overline{\Delta G}_{\mathrm{In}}^{\mathrm{ex}}$ & & & $a_{\text {In }}$ & & $\Delta H^{19}$ & \\
\hline 0,01 & $-24,14$ & 0 & $-0,25$ & $5 \cdot 10^{-8}$ & 0,99 & $-0,10$ & $+0,14$ \\
\hline 0,02 & $-22,82$ & $-0,02$ & $-0,47$ & $2 \cdot 10^{-7}$ & 0,97 & $-0,21$ & $+0,26$ \\
\hline 0,05 & $-18,82$ & $-0,16$ & $-1,09$ & $3,8 \cdot 10^{-6}$ & 0,88 & $-0,48$ & $+0,61$ \\
\hline 0,10 & $-13,31$ & $-0,61$ & $-1,88$ & $1,2 \cdot 10^{-4}$ & 0,66 & $-0,94$ & $+0,94$ \\
\hline 0,15 & $-7,58$ & $-1,42$ & $-2,34$ & $3,3 \cdot 10^{-3}$ & 0,37 & $-1,33$ & $+1,01$ \\
\hline 0,18 & $-4,48$ & $-2,03$ & $-2,47$ & $1,9 \cdot 10^{-2}$ & 0,22 & $-1,51$ & $+0,96$ \\
\hline
\end{tabular}

Tab. 6. Thermodynamische Bildungswerte fester $\alpha$-Mischphasen des Silber-Germanium- und Silber-Zinn-Systems bei $1000^{\circ} \mathrm{K}$. $\Delta G$. und $\Delta H$-Werte in $\mathrm{kcal} / \mathrm{g}$-Atom und $\Delta S$.Werte in cal $/ \mathrm{g}$-Atom ${ }^{\circ} \mathrm{K}$.

\begin{tabular}{|c|c|c|c|c|c|c|c|}
\hline$x_{\mathrm{Ge}}$ & $\overline{\Delta G}_{\mathrm{Ge}}^{\mathrm{ex}}$ & $\overline{\Delta G}_{\mathrm{Ag}}^{\mathrm{ex}}$ & $\Delta G \mathrm{ex}$ & $a_{\mathrm{Ge}}$ & $a_{\mathrm{Ag}}$ & $\Delta H^{20}$ & $\Delta S^{\mathrm{ex}}$ \\
\hline 0,01 & $-25,91$ & $-0,01$ & $-0,27$ & $2 \cdot 10^{-8}$ & 0,98 & & \\
\hline 0,02 & $-23,14$ & $-0,04$ & $-0,51$ & $1,7 \cdot 10^{-7}$ & 0,96 & $+0,65$ & $+1,15$ \\
\hline 0,03 & $-19,52$ & $-0,13$ & $-0,71$ & $1,6 \cdot 10^{-6}$ & 0,91 & & \\
\hline 0,04 & $-16,22$ & $-0,25$ & $-0,89$ & $1,1 \cdot 10^{-5}$ & 0,85 & $+0,52$ & $+1,41$ \\
\hline 0,05 & $-14,35$ & $-0,34$ & $-1,04$ & $3,1 \cdot 10^{-5}$ & 0,80 & & \\
\hline 0,06 & $-12,22$ & $-0,47$ & $-1,17$ & $1,3 \cdot 10^{-4}$ & 0,74 & $+0,75$ & $+1,92$ \\
\hline 0,07 & $-10,32$ & $-0,59$ & $-1,27$ & $3,9 \cdot 10^{-4}$ & 0,69 & & \\
\hline$x_{\mathrm{Sn}}$ & $\overline{\Delta G}_{\mathrm{sn}}^{\mathrm{ex}}$ & & & $a_{\mathrm{Sn}}$ & & $\Delta H^{21}$ & \\
\hline 0,01 & $-26,47$ & 0 & $-0,27$ & $2 \cdot 10^{-8}$ & 0,99 & $-0,05$ & $+0,22$ \\
\hline 0,02 & $-24,99$ & $-0,02$ & $-0,52$ & $9 \cdot 10^{-8}$ & 0,97 & $-0,12$ & $+0,30$ \\
\hline 0,04 & $-22,40$ & $-0,10$ & $-0,99$ & $5,1 \cdot 10^{-7}$ & 0,92 & $-0,27$ & $+0,72$ \\
\hline 0,06 & $-21,08$ & $-0,17$ & $-1,42$ & $1,5 \cdot 10^{-6}$ & 0,87 & $-0,39$ & $+1,03$ \\
\hline 0,08 & $-19,89$ & $-0,26$ & $-1,83$ & $3,6 \cdot 10^{-6}$ & 0,81 & $-0,51$ & $+1,32$ \\
\hline 0,10 & $-19,29$ & $-0,22$ & $-2,13$ & $5,9 \cdot 10^{-6}$ & 0,82 & $-0,62$ & $+1,51$ \\
\hline 0,11 & $-18,50$ & $-0,19$ & $-2,20$ & $9,9 \cdot 10^{-6}$ & 0,81 & $-0,68$ & $+1,52$ \\
\hline
\end{tabular}

Die folgenden Betrachtungen sollen an Hand der Differenz der Überschußwerte der partiellen freien Mischungsenthalpien beim Entfernen eines Atoms des Wirtsgitters und gleichzeitigem Hinzufügen eines Fremdatoms vorgenommen werden. Diese Differenz setzt sich aus den genannten drei Anteilen zusammen:

$$
\begin{aligned}
\overline{\Delta G}_{\mathrm{A}}^{\mathrm{ex}}-\overline{\Delta G}_{\mathrm{Ag}}^{\mathrm{ex}}=\left(\overline{\Delta G}_{\mathrm{A}}^{\mathrm{V}}-\overline{\Delta G}_{\mathrm{Ag}}^{\mathrm{V}}\right) \\
+\left(\overline{\Delta G}_{\mathrm{A}}^{\mathrm{U}}-\overline{\Delta G}_{\mathrm{Ag}}^{\mathrm{U}}\right)+\left(\overline{\Delta G}_{\mathrm{A}}^{\mathrm{B}}-\overline{\Delta G}_{\mathrm{Ag}}^{\mathrm{B}}\right) .
\end{aligned}
$$

V kennzeichnet den Verzerrungsanteil, U den Umwandlungsanteil und B den Bindungsanteil. Es sei zunächst der Verzerrungsanteil betrachtet.

\section{Abschätzung des Gitterverzerrungseinflusses}

Beim Einbau eines größeren oder kleineren Fremdatoms in ein Wirtsgitter wird das Gitter in der Umgebung des Fremdteilchens verzerrt. Es ist eine entsprechende „Lochbildungsenergie“ $E_{\mathrm{L}}$ aufzubringen. Diese Verzerrung wird in gewissem Umfang reduziert, wenn zwei solcher Verzerrungszentren benachbart auftreten. Beim Zusammentreten isolierter Verzerrungszentren zu Komplexen mit je zwei benachbarten Fremdatomen kann gewissermaßen eine „Assoziationsenergie“ $w$ gewonnen werden. Nach BRODOWSKY ${ }^{2}$ kann unter Anwendung der quasi-chemischen Näherung ${ }^{22}$ der Verzerrungsanteil in folgen- 
der Weise abgeschätzt werden:

$$
\overline{\Delta G}_{\mathrm{A}}^{\mathrm{V}}-\overline{\Delta G}_{\mathrm{B}}^{\mathrm{V}}=E_{\mathrm{L}}+Z R T \ln \frac{2\left(1-x_{\mathrm{A}}\right)}{1-2 x_{\mathrm{A}}+\beta}
$$

mit

$$
\beta:=\left\{1-4 x_{\mathrm{A}}\left(1-x_{\mathrm{\Lambda}}\right)\left[1-\exp \left(-\frac{w}{R T}\right)\right]\right\}^{1 / 2} .
$$

$Z$ ist die Koordinationszahl des Wirtsgitters. Für $x_{\mathrm{A}}=0$ wird $\overline{\Delta G}_{\mathrm{A}}^{\mathrm{V}}-\overline{\Delta G}_{\mathrm{B}}^{\mathrm{V}}=E_{\mathrm{L}}$. Für ein kubisch flächenzentriertes Gitter ist ${ }^{2}$ :

$$
E_{\mathrm{L}}=-6 w \text {. }
$$

Zur Abschätzung von $E_{\mathrm{L}}$ kann nach HeUmanN ${ }^{5}$ die Volumenabhängigkeit der inneren Energie des Systems unter Zugrundelegung eines elastischen Gittermodells herangezogen werden. Näherungsweise gilt

$$
E_{\mathrm{L}}=T \frac{\alpha_{\mathrm{B}}}{\varkappa_{\mathrm{B}}} \Delta V_{\mathrm{B}}+\frac{\left(\Delta V_{\mathrm{B}}\right)^{2}}{2 V_{\mathrm{B}} \varkappa_{\mathrm{B}}} .
$$

$\alpha_{\mathrm{B}}$ bedeutet den kubischen thermischen Ausdehnungskoeffizienten und $\varkappa_{\mathrm{B}}$ die isotherme Kompressibilität der reinen Wirtskomponente. $\Delta V_{\mathrm{B}}$ stellt die Differenz der Atomvolumina der Komponenten A und B dar. Nach HeumanN ${ }^{5}$ ist $T=0,4 T_{\mathrm{s}}$ zu setzen, wobei $T_{\mathrm{s}}$ die absolute Schmelztemperatur der Wirtskomponente ist.

Nach Gl. (17) wurden für die hier interessieren- . den Systeme die Verzerrungsanteile abgeschätzt. An Stelle der Atomvolumendifferenz der Mischungspartner wurde die aus röntgenographischen Bestimmungen der Gitterkonstanten gegebene reale $\mathrm{Zu}$ nahme des Atomvolumens der Wirtskomponente beim Zulegieren einer Fremdkomponente verwendet. Die Gitterkonstanten wurden dem Sammelwerk von PeArson ${ }^{23}$ entnommen. Die resultierenden $E_{\mathrm{L}^{-}}$ Werte sind in Tab. 7 zusammengestellt.

Tab. 7. Abschätzung der Lochbildungsenergie $E_{\mathrm{L}}$ fester Silber-Mischphasen nach Gl. (17).

\begin{tabular}{llll}
\hline Metall B & $\begin{array}{l}V_{\mathrm{B}} \\
{\left[\mathrm{cm}^{3} / \mathrm{g}-\mathrm{At} .\right]}\end{array}$ & $\begin{array}{l}\alpha_{\mathrm{B}} \\
{\left[\left({ }^{\circ} \mathrm{K}\right)^{-1}\right]}\end{array}$ & $\begin{array}{l}x_{\mathrm{B}} \\
{\left[\mathrm{cm}^{3} / \mathrm{cal}\right]}\end{array}$ \\
\hline $\mathrm{Ag}^{24}$ & 10,27 & $57,0 \cdot 10^{-6}$ & $32,4 \cdot 10^{-6}$ \\
\hline System & $\begin{array}{l}\Delta a_{\mathrm{B}}{ }^{23} \\
{[\% / \mathrm{g}-\mathrm{At} .]}\end{array}$ & $\begin{array}{l}\Delta V_{\mathrm{B}} \\
{\left[\mathrm{cm}^{3} / \mathrm{g}-\mathrm{At} .\right]}\end{array}$ & $\begin{array}{l}E_{\mathrm{L}} \\
{[\mathrm{kcal} / \mathrm{g} \text {-At. }]}\end{array}$ \\
\hline $\mathrm{Ag}-\mathrm{Au}$ & $+0,24$ & $+0,07$ & 0 \\
$\mathrm{Ag}-\mathrm{Ga}$ & $-1,65$ & $+0,51$ & 0 \\
$\mathrm{Ag}-\mathrm{Ge}$ & $+0,69$ & $+0,01$ & 0 \\
$\mathrm{Ag}-\mathrm{Cd}$ & $+5,30$ & $+1,63$ & $+4,1$ \\
$\mathrm{Ag}-\mathrm{In}$ & $+7,67$ & $+2,36$ & $+8,0$ \\
$\mathrm{Ag}-\mathrm{Sn}$ & $+10,50$ & $+3,24$ & $+14,3$ \\
\hline
\end{tabular}

Es sei noch bemerkt, daß die unter Zugrundelegung eines einfachen elastischen Modells gewonnenen $E_{\mathrm{L}}$-Werte mit steigender Temperatur abnehmen sollten. Im Hinblick auf unsere relativ hohen Versuchstemperaturen dürften die in Tab. 7 aufgeführten Zahlenwerte für $E_{\mathrm{L}}$ daher obere Grenzwerte darstellen. Die Unsicherheit bei der Abschätzung des Verzerrungsbeitrags wird jedoch in den meisten Fällen nur einen geringen Fehler bei der Ermittlung des elektronischen Bindungsanteils nach Gl. (14) zur Folge haben, da der Verzerrungsbeitrag in unserem Falle meist nur eine relativ kleine Korrektur der gemessenen Überschußfunktionen darstellt.

\section{Abschätzung der Zustandsdichte des Leitungs- bandes des Silbers an der Fermi-Grenze}

Wird vorerst ein möglicher Umwandlungsbeitrag außer acht gelassen, dann resultieren gemäß Gl. (14) unmittelbar die Bindungsanteile $\left.\overline{(\Delta G}_{\mathrm{A}}^{\mathrm{B}}-\overline{\Delta G}_{\mathrm{B}}^{\mathrm{B}}\right)$.

Das Leitungsband des Silbers (entsprechend dem Zustand $5 s^{1}$ ) ist bekanntlich nur halb mit Elektronen gefüllt. Bei der Substitution von Silberatomen durch Indium- oder Zinn- bzw. Gallium- und Germanium-Atome wird das Fremdatom mit dem Elektronenzustand des Silbers $4 \mathrm{~d}^{10} 5 \mathrm{~s}^{1}$ bzw. einem ähnlichen Elektronenzustand $3 \mathrm{~d}^{10} 4 \mathrm{~s}^{1}$ in die Grundmatrix des Silbergitters eingebaut. Die restlichen äußeren Elektronen werden zu einem Teil abgeschirmt und zum anderen Teil in das Leitungsband des Silbers abgeführt und zum Anheben des FermiNiveaus verwendet. Dieser elektronische Auffülleffekt sollte sich im Betrag des Bindungsanteils der partiellen freien Bildungsenthalpie bemerkbar machen.

Der Auffülleffekt je Fremdatom hängt naturgemäß von der Wertigkeitsdifferenz zwischen den Legierungspartnern ab. Es ist daher zweckmäßig, den Bindungsanteil nicht als Funktion des Legierungsgehaltes, sondern in Abhängigkeit von einer effektiven Konzentration

$$
x_{\mathrm{A}}^{\text {eff }}:=\left(z_{\mathrm{A}}-z_{\mathrm{Ag}}\right) x_{\mathrm{A}}
$$

zu betrachten. In Abb. 4 ist der Zusammenhang graphisch dargestellt.

Bei der Auftragung des Bindungsanteils $\left(\overline{\Delta G}_{\mathrm{A}}^{\mathrm{B}}\right.$ $-\overline{\Delta G}_{\mathrm{Ag}}^{\mathrm{B}}$ ) gegen die effektive Konzentration der Legierungspartner sollte, sofern der Bindungseffekt ausschließlich von einer ungestörten Auffüllung des Leitungsbandes des Silbers herrührt, ein linearer 


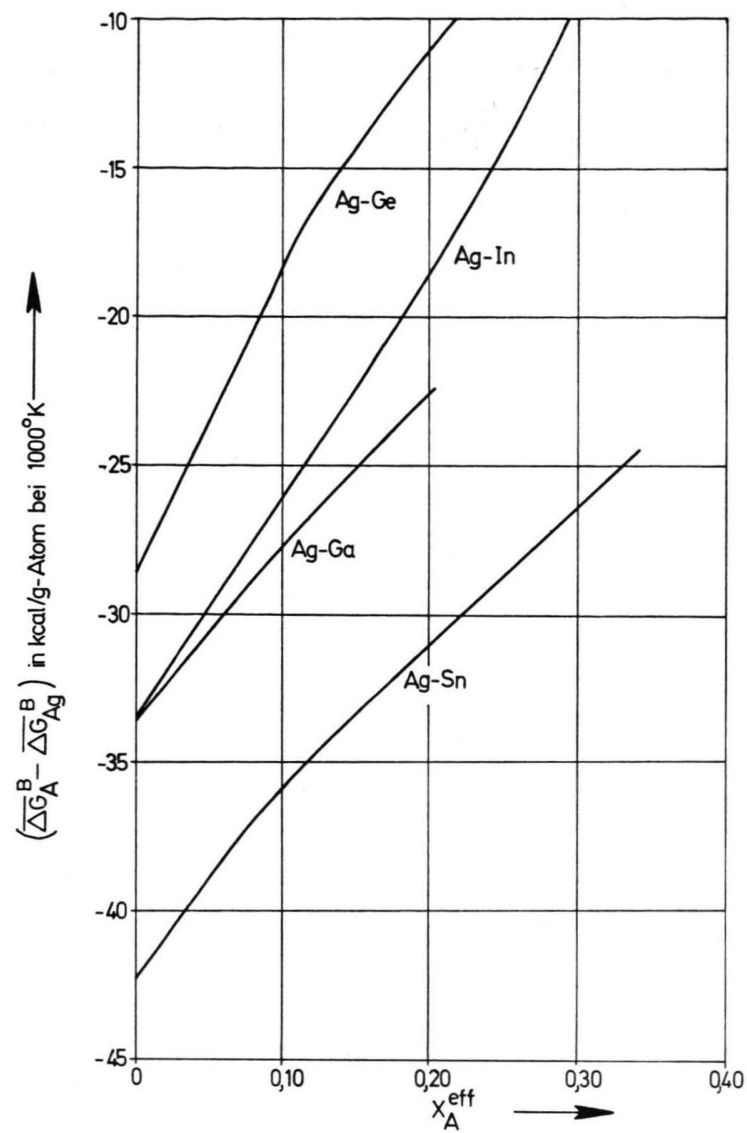

Abb. 4. Verlauf der Bindungsbeiträge der partiellen freien Bildungsenthalpie mit der effektiven Konzentration für a-Silber-Mischkristalle bei $1000^{\circ} \mathrm{K}$.

Zusammenhang erwartet werden. Die geringsten Störungen der Bandstruktur durch den Einbau fremder Ionenrümpfe ist bei geringer Fremdatomkonzentration zu erwarten. In Abb. 5 sind die Bindungsanteile bei unendlicher Verdünnung gegen die Wertigkeitsdifferenz der Komponenten aufgetragen. Wie unmittelbar ersichtlich, ist die erwartete Linearität, wenn von dem $\overline{\Delta G_{\mathrm{A}}^{\mathrm{B}}, x_{\mathrm{A}=0}}$-Wert für die $\mathrm{Ag}-\mathrm{Ge}$ Mischkristalle abgesehen wird, tatsächlich erfüllt. Auch der für $\mathrm{Ag}-\mathrm{Au}$ - und $\mathrm{Ag}-\mathrm{Cd}$-Mischkristalle aus Literaturdaten ermittelte Bindungsanteil ordnet sich in diesen Zusammenhang zwanglos ein ${ }^{26,27}$.

Die erhebliche Abweichung des „Bindungsanteils" des verdünnten $\mathrm{Ag}-\mathrm{Ge}-$ Mischkristalls von der sonst erfüllten linearen Abhängigkeit in Abb. 5 dürfte zweifellos auf einen im $\left(\overline{\Delta G}_{\mathrm{A}}^{\mathrm{B}}-\overline{\Delta G}_{\mathrm{Ag}}^{\mathrm{B}}\right)$-Wert noch enthaltenen, beträchtlichen Umwandlungsanteil zurückzuführen sein. Offensichtlich ist der Übergang des Germaniums von seiner Diamantstruktur mit

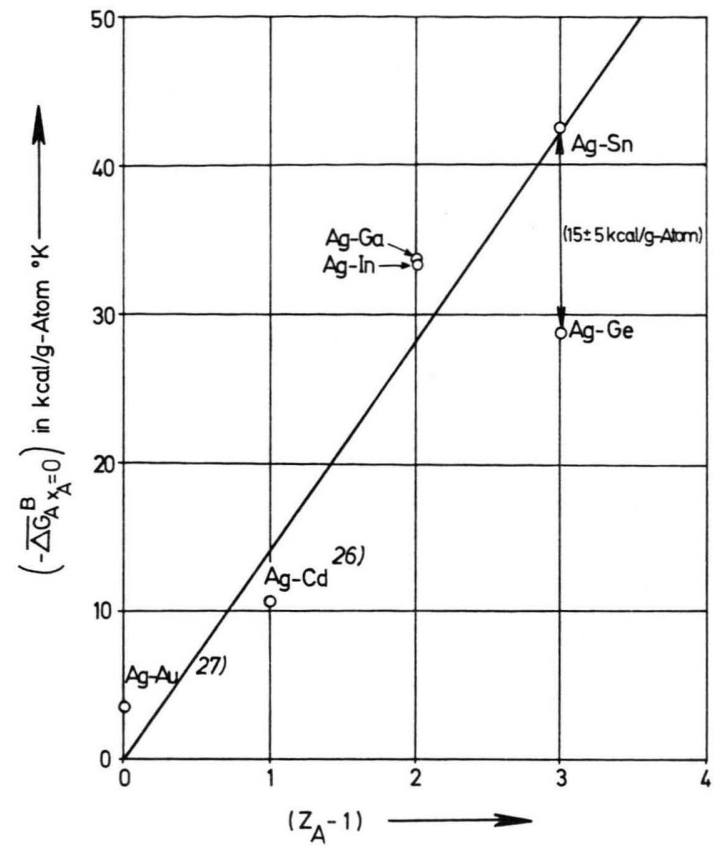

Abb. 5. Abhängigkeit der Bindungsbeiträge der partiellen freien Bildungsenthalpie für unendliche Verdünnung von der Wertigkeitsdifferenz der Legierungspartner für $\alpha$-Silber-Mischkristalle bei $1000^{\circ} \mathrm{K}$. In der Beschriftung der Ordinatenachse muß es „bei $1000^{\circ} \mathrm{K}$ “ heißen.

homöopolaren Bindungen in eine kubisch flächenzentrierte Struktur mit freien Elektronen mit einem erheblichen Aufwand an freier Enthalpie verbunden. Auch im Falle des Galliums, Indiums und Zinns wären bei der oben durchgeführten Betrachtung noch Umwandlungsanteile $\left(\overline{\Delta G}_{\mathrm{A}}^{\mathrm{U}}-\overline{\Delta G}_{\mathrm{Ag}}^{\mathrm{U}}\right)$ zu berücksichtigen. Diese sind aber zweifellos erheblich kleiner als im Falle des Germaniums und treten offenbar nicht über die Fehlergrenzen der erhaltenen

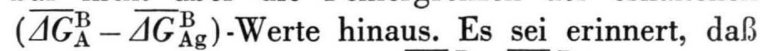
die zur Bestimmung der $\left(\overline{\Delta G}_{\mathrm{A}}^{\mathrm{B}}-\overline{\Delta G}_{\mathrm{Ag}}^{\mathrm{B}}\right)$-Werte benutzten Verzerrungsanteile, $\left(\overline{\Delta G}_{\mathrm{A}}^{\mathrm{V}}-\overline{\Delta G}_{\mathrm{Ag}}^{\mathrm{V}}\right)$, lediglich als Schätzwerte anzusehen sind und eine gewisse Unsicherheit in die hier betrachteten Werte der Bindungsanteile einbringen. Aus der Abweichung des

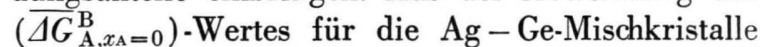
von der in Abb. 5 eingezeichneten mittelnden Geraden ergibt sich der Umwandlungsbeitrag des Germaniums zu

$$
\left(\overline{\Delta G}_{\mathrm{Ge}, x \mathrm{Ge}=0}^{\mathrm{u}}\right)=+15 \pm 5 \mathrm{kcal} / \mathrm{g} \text {-Atom . }
$$

Es scheint in gewissem Umfang möglich zu sein, aus dem Gang des Bindungsanteils mit der effektiven Konzentration auf Eigenheiten des Leitungsbandes zu schließen. Zumindest ist dies bei kleinen Kon- 
zentrationen erlaubt, bei denen keine nennenswerte Änderung der Bandstruktur durch die Fremdionenrümpfe zu erwarten ist. Der Kehrwert der Steigung der Kurven der Abb. $4\left[\mathrm{~d}\left(\overline{\Delta G}_{\mathrm{A}}^{\mathrm{B}}-\overline{\Delta G}_{\mathrm{Ag}}^{\mathrm{B}}\right) / \mathrm{d} x_{\mathrm{A}}^{\text {eff }}\right]$ hat die physikalische Bedeutung der Zustandsdichte $\mathrm{d} n / \mathrm{d} E$ des Leitungsbandes des Silbers. Eine Zunahme der effektiven Konzentration um $\Delta x_{A}^{\text {eff }}$ beim Zulegieren der höherwertigen Komponente führt zu einer Erhöhung der Fermi-Energie $\Delta E_{\mathrm{F}}$ des Leitungsbandes der einwertigen Grundkomponente. Aus der Anfangssteigung der $\left(\overline{\Delta G}_{\mathrm{A}}^{\mathrm{B}}-\overline{\Delta G}_{\mathrm{Ag}}^{\mathrm{B}}\right)-x_{\mathrm{A}}^{\text {eff }}$-Kurven bei $x_{A}^{\text {eff }}=0$ (vgl. Abb. 4) ergeben sich folgende Werte für die Zustandsdichten des Leitungsbandes des Silbers an der Fermi-Grenze $E_{\mathrm{F}}$ :

Aus dem System

$$
\begin{array}{ll}
\mathrm{Ag}-\mathrm{Ga}: & 0,38 \\
\mathrm{Ag}-\mathrm{In}: & 0,31 \\
\mathrm{Ag}-\mathrm{Ge}: & 0,23 \\
\mathrm{Ag}-\mathrm{Sn}: & \mathbf{0 , 3 6}
\end{array}
$$

in Elektronen/Atom und $\mathrm{eV}$.

Bei dieser Auswertung wurde Abschirmungseffekten ${ }^{24 a}$ nicht Rechnung getragen, so daß die $x_{A}^{\text {eff }}$-Werte nach Gl. (18) zu groß angesetzt sind. Dementsprechend dürften die hier ermittelten Beträge für die Zustandsdichte des Silbers lediglich obere Grenzwerte darstellen. Eine quantitative Behandlung des Abschirmeffektes scheint zur Zeit für die hier interessierenden Fälle noch recht problematisch zu sein. Ein Vergleich der $n\left(E_{\mathrm{F}}\right)$-Werte mit dem aus photoelektrischen Messungen an Silber von BERGLUND und SPICER ${ }^{25}$ erhaltenen Wert von 0,37 Elektronen/Atom und $\mathrm{eV}$ zeigt eine gute Übereinstimmung. Messungen von Elektronenwärmen, von CORAK und Mitarb. ${ }^{34}$ durchgeführt, ergaben kleinere $n\left(E_{\mathrm{F}}\right)$ Werte von 0,17 Elektronen/Atom und eV. Theoretische Rechnungen von SNOw ${ }^{35}$ und BHATNAGAR ${ }^{28}$ lieferten Werte von 0,28 bzw. 0,37 Elektronen/Atom und $\mathrm{eV}$ für Silber.

Es sei noch erwähnt, daß die erzielte Zuverlässigkeit der von uns erhaltenen Werte für die Zustandsdichte in erster Linie auf der Genauigkeit der mit der Kette (5) erzielten experimentellen Resultate beruht. Durch Absolutmessungen der $\overline{\Delta G}_{\mathrm{A}}$-Werte allein mittels der Kette (3) resultieren wesentlich weniger zuverlässige Steigmaße für die $\overline{\Delta G}_{\mathrm{A}}-x_{\mathrm{A}}$-Funktion bei sehr kleinen Konzentrationen $x_{\mathrm{A}}$, die meist zu niedrige Zustandsdichten vortäuschen ${ }^{29}$.

Wie aus Abb. 5 ersichtlich, nimmt die Steigung $\operatorname{der}\left(\overline{\Delta G}_{\mathrm{A}}^{\mathrm{B}}-\overline{\Delta G}_{\mathrm{Ag}}^{\mathrm{B}}\right)$-Kurven mit der effektiven Kon- zentration $x_{A}^{\text {eff }}$ ab. Das könnte sowohl auf die Vernachlässigung des Abschirmeffektes als auch auf die Änderung der Zustandsdichte mit der Elektronenkonzentration in den hier untersuchten $\alpha$-Mischphasen des Silbers zurückzuführen sein. Der Charakter der Steigmaßänderung ist abhängig von der Natur der Fremdkomponente. Offenbar ist die strenge Gültigkeit des Modells „starrer" Bänder ${ }^{24}$ hier nicht mehr gegeben. Möglicherweise beeinflussen die verschiedenen Fremdkomponenten der Silber-Mischkristalle die Gestalt der Fermi-Oberfläche des Silbers ${ }^{30,31}$ derart, daß die „Bauchpartie“ zunimmt und die den Kontakt mit der benachbarten Fermi-Oberfläche herstellende „Halspartie“ abnimmt.

Nach KING und Massalski ${ }^{32}$ dürfte eine bei der Legierungsbildung auftretende Verminderung der s-d-Überlappung im Elektronenband des Silbers - anders als im Falle des Kupfers bzw. Goldes nur einen vernachlässigbaren Änderungseffekt ${ }^{33}$ auf die Bandstruktur bewirken.

\section{Erörterung der Überschußentropien}

Zur Diskussion der Überschußwerte der Bildungsentropien der Silber-Mischkristalle kann man ebenfalls eine Aufteilung in Einzelbeträge durchführen, und zwar im einfachsten Falle in einen Anteil, der durch die Änderung des Gitterschwingungsspektrums bedingt ist, und einen weiteren Anteil, der durch die Änderung der Elektronenwärme zustande kommt:

$$
\Delta S^{\mathrm{ex}}=\int \frac{\Delta c_{\mathrm{p}}^{\text {Gitter }}}{T} \mathrm{~d} T+\int \frac{\Delta c_{\mathrm{p}}^{\text {Elektr. }}}{T} \mathrm{~d} T .
$$

Beim Einbau der Fremdkomponenten Indium, Zinn, Gallium bzw. Germanium in das Silberwirtsgitter bringen diese Fremdkomponenten den gleichen $5 \mathrm{~s}^{1}$ Elektronen-Zustand wie das Silber bzw. den gleichwertigen $4 \mathbf{s}^{1}$-Zustand in das Wirtsgitter ein. Es sollte deswegen erwartet werden, daß sich dabei das Schwingungsspektrum des Gitters nur unwesentlich ändert. Der Überschußwert der Bildungsentropie sollte sich letztlich wesentlich aus einer Änderung der Elektronenwärme bei der Legierungsbildung ergeben. Betrachtet man die Konzentrationsabhängigkeit der Bindungsfunktionen in Abb. 4 für die Silbermischphasen und die aus der thermodynamischen Auswertung der Silbersysteme erhaltenen Überschußwerte für die Bildungsentropie in Tab. 5 und 6, so fällt auf, daß die Systeme mit relativ großen Überschußentropiewerten auch starke Verände- 
rungen der Steigung der Bindungsfunktionen mit der Konzentration zeigen. Dies könnte darauf hindeuten, daß die positiven Überschußwerte der Bildungsentropie und diese Steigmaßänderung die gleiche Ursache haben, und zwar eine beim Einbau von Fremdteilchen auftretende Veränderung der FermiOberfläche im Wirtsgitter. Dadurch nimmt die Elektronenwärme der Leitungselektronen zu. Dies verursacht dann einen positiven Überschußwert der Bildungsentropien der festen Mischphasen.

1 Th. Heumann u. B. Predel, Z. Metallkde. 51, 509 [1960].

2 H. Brodowsky, Z. Naturforsch. 22 a, 130 [1967].

3 O. Alpaut u. Th. Heumann, Acta Met. 13, 543 [1965].

4 A. Lawson, J. Chem. Physics 15, 831 [1947].

5 Th. Heumann, Z. Elektrochem. 57, 724 [1953].

6 B. Predel, Acta Met. 14, 209 [1966].

7 B. Predel u. W. Schwermann, Z. Naturforsch. 23 a, 2052 [1968].

8 R. A. Rapp u. F. MaAK, Acta Met. 10, 63 [1962].

9 H. Brodowsky, Z. Phys. Chem. N.F. 44, 129 [1965].

10 A. W. Bryant, W. G. Bugden u. J. N. Pratt, Acta Met. 18, 101 [1970].

11 H. BRodowsky u. H. J. Schaller, demnächst.

12 K. Kiukkola u. C. WAgner, J. Electrochem. Soc. 104, 379 [1957].

13 H. Schmalzried, Z. Phys. Chem. 25, 178 [1960].

14 C. H. Steele u. C. B. Alcock, Trans. Met. Soc. AIME 233, 1359 [1965].

15 R. C. WEAST, Handbook of Chemistry and Physics, 49th Edition, Cleveland, Ohio 1968/69.

16 F. Hund u. R. Mezger, Z. Phys. Chem. 201, 268 [1952].

17 P. C. Lidster u. H. B. Bell, Trans. Met. Soc. AIME 245, 2273 [1969].

18 B. Predel u. D. W. Stein, Acta Met. 20, 515 [1972].

19 O. J. Kleppa, J. Phys. Chem. 60, 846 [1956].

20 B. Predel u. D. W. Stein, Z. Naturforsch. 26 a, 722 [1971].
Herrn Prof. Dr. Th. HeumanN danken wir für die wohlwollende Förderung dieser Arbeit. - Herrn Prof. Dr. H. Brodowsky und Herrn Dr. H. J. Schaller sind wir für klärende Diskussionen und wertvolle Hinweise zu Dank verpflichtet. - Unser Dank gilt ferner dem Landesamt für Forschung des Landes NordrheinWestfalen und dem Fonds der Chemischen Industrie für die Bereitstellung von Forschungsmitteln.

21 O. J. Kleppa, Acta Met. 3, 255 [1955].

22 T. L. Hill, Introduction to Statistical Thermodynamics. Reading, London 1960, p. 252.

23 W. B. Pearson, A Handbook of Lattice Spacings and Structures of Metals and Alloys, Pergamon Press, London, New York, Paris, Los Angeles, Vol. I u. II, 1958 u. 1967.

${ }^{24}$ N. F. MotT u. H. Jones, The Theory of the Properties of Metals and Alloys, Oxford 1936.

${ }^{24 a}$ J. Friedel, Phil. Mag. Suppl. 45, 456 [1954]

25 C. N. Berglund u. W. E. Spicer, Phys. Rev. 136, A 1044 [1964].

26 T. B. Massalski u. H. W. King, Progress in Materials Science 10, 19 [1961], Pergamon Press.

27 M. H. Cohen u. V. Heine, Adv. Phys. 7, 395 [1958].

28 S. Bhatnagar, Phys. Rev. 183, 657 [1969].

29 B. Predel u. U. Schallner, Proceedings of the Symposium on Metallurgical Chemistry, Brunel University, Uxbridge, England, July 1971, im Druck.

30 D. Shoenberg, Nature London 183, 171 [1959]; Phil. Mag. 5, 105 [1960].

31 R. W. Morse, A. Myers u. C. T. Walter, Phys. Rev. Let ters 4, 605 [1960].

32 W. S. Corak, M. P. Garfunkel, C. B. Satterthwaite u. A. WeXler, Phys. Rev. 98, 1699 [1955].

33 E. C. Snow, Phys. Rev. 172, 708 [1968]. 